\title{
The Effect of an Arthroscopic Orthopaedic Procedure on a Professional Tennis Player's Career
}

\author{
Andrew George ${ }^{1}$, Matthew D. Saltzman ${ }^{1}$, Wellington K. Hsu ${ }^{1}$ \\ 1. Orthopaedic Surgery, Northwestern University Feinberg School of Medicine, Chicago, USA
}

Corresponding author: Andrew George, andrew.george@northwestern.edu

\begin{abstract}
Orthopaedic injuries can significantly impact the careers of professional tennis players. It is currently unknown how professional tennis players fare after arthroscopic surgery. For the purpose of this study, players ranked in the Association of Tennis Professionals (ATP) and Women's Tennis Association (WTA) who underwent arthroscopic surgery of any joint between 1996 and 2016 were identified through a wellestablished, previously published protocol of injury reports and public archives. Performance statistics both before and after surgery, time to return (TTR) to play, and career length following surgery were collected for each player. Statistical analysis was performed with significance accepted at a probability value (p) of $<0.05$. A total of 55 (39 males and 16 females) players met the inclusion criteria (shoulder, $\mathrm{n}=15$; elbow, $\mathrm{n}=15$; wrist, $\mathrm{n}=13$; hip, $\mathrm{n}=12)$. The average age of the players at the time of surgery was $25.8( \pm 4)$ years, and the average career length before surgery was $8.4( \pm 4)$ years. Tennis players who underwent arthroscopic shoulder surgery experienced a longer TTR to play ( 279 days, $\mathrm{p}<0.01$ ), as well as a greater decline in their rankings, both in the first and second years postoperatively ( $<<0.01$ and $p=0.01$, respectively), compared to all other surgical cohorts. Players who underwent surgery on the elbow, wrist, and hip had no significant decline in the ranking by the second postoperative year. There were no significant differences between genders. This study represents the largest database of professional tennis players who have undergone arthroscopic surgery and may allow physicians to provide evidence-based recommendations about expectations after surgical treatment.
\end{abstract}

Categories: Orthopedics

Keywords: tennis, surgery, outcomes

\section{Introduction}

The physical demands of professional tennis players often lead to a high rate of musculoskeletal injuries [13]. Modern racquets have enabled players to generate ball velocities upwards of $140 \mathrm{mph}$, resulting in a substantial amount of force transmitted through the body at contact with the ball $[4,5]$. Furthermore, the force used to generate these ball velocities flows through a kinetic chain: from the lower extremities through the trunk, and ultimately into the shoulder, elbow, and wrist [6]. Structural damage and injury to ligamentous, tendinous, and labral structures can occur as a result of repetitive forces experienced by these joints. The emphasis on ball velocity, along with very demanding professional tour schedules, has been associated with an increase in injuries over the past 20 years, resulting in a higher rate of injury per playing hour in tennis compared to other sports such as rugby and basketball [1-3].

Arthroscopic surgery is often considered for structural injuries when conservative treatment fails. Prior studies have demonstrated favorable outcomes after surgery in recreational tennis players and in other overhead athletes such as baseball pitchers [7-12], but few studies have critically examined outcomes in professional tennis players. Since professional tennis players have substantially greater physical demands than recreational athletes, their return-to-play and career-length outcomes may vary. While small case series have reported limited performance statistics after individual procedures [13,14], their impact on the professional player's career in terms of short-, medium-, and long-term consequences is unknown. It is also unclear how outcomes differ depending on which joint is affected. This information may be helpful for players, coaches, and physicians assessing the potential impact of an injury and surgery on a professional tennis player's career.

In our study, we sought to assess and compare performance-based outcomes following arthroscopic surgery in both male and female professional tennis players by utilizing important measures including world ranking, recovery time, and career length.

\section{Materials And Methods}

Using a previously published methodology that involved the review of public archives [15-20], we identified professional singles tennis players ranked in the Association of Tennis Professionals (ATP) and Women's Tennis Association (WTA) who underwent arthroscopic surgery of any joint between 1996 and 2016. 
Institutional review board approval was not required for this study as it used public data only. Online databases including NewsBank (NewsBank Inc., Naples, FL), the Infosys ATP Player Database, and the WTA Player Database were explored using search terms such as "ATP injury", "WTA injury", "professional tennis injury”, "arthroscopic surgery”, "shoulder surgery”, "elbow surgery”, “wrist surgery”, "hip surgery”, "knee surgery", and "ankle surgery". Players were deemed to meet the inclusion criteria if reports of the injury, surgery, and date of surgery were corroborated by at least two independent sources of information, including press releases, newspaper archives, and injury reports. Players were excluded if they had undergone concurrent procedures, had received only non-operative management, or had undergone revision procedures within 3 years of surgery. Those players whose reports contained conflicting information from different sources were also excluded. In addition, players who primarily played in doubles matches were excluded, due to potential variability in doubles partner skills and performance. The shoulder, elbow, wrist, and hip cohorts were included, as their sample sizes met the threshold for statistical analysis using a oneway independent analysis of variance $(n=10, a=0.05, b=0.2)$.

Player variables including age, sex, body mass index (BMI), hand dominance, career length before surgery, date of surgery, date of the first match played after surgery, and career length after surgery were collected for each athlete. To assess performance, we collected year-end singles ranking in the preoperative year, as well as the rankings in the first and second years postoperatively. In addition, we collected the number of aces per service game for male players (not available for female players) using the Infosys ATP Player Database. The preoperative year was defined as the year immediately preceding the injury-shortened year. Postoperative years one and two were defined as the first and second years after the athlete returned postsurgery, respectively The athlete's preoperative performance was used as a baseline to compare with postoperative performance. We did not include data beyond the postoperative year two due to the potential effects of confounding variables such as unrelated injuries, age, and talent. Finally, time to return (TTR) was defined as the number of days between surgery and the first match played postoperatively.

Statistical analysis was performed using Statistical Analysis System (SAS; version 9.4; SAS Institute, Cary, NC). Cohorts of players undergoing arthroscopic shoulder, elbow, wrist, and hip surgery were compared using analysis of variance (ANOVA). Each athlete served as his/her own control with a two-tailed paired ttest used to evaluate changes in performance after surgery. Statistical analysis was performed with significance accepted at a probability value (p) of $<0.05$.

\section{Results}

A total of 55 (39 males and 16 females) professional tennis players who underwent arthroscopic surgery between 1996 and 2016 were included (shoulder, $\mathrm{n}=15$; elbow, $\mathrm{n}=15$; wrist, $\mathrm{n}=13$; hip, $\mathrm{n}=12$ ) (Table 1). Although reported diagnoses leading to surgical intervention included rotator cuff tear, labral tear of the shoulder, bone spurs of the elbow, ligament damage of the wrist, and femoroacetabular impingement of the hip, this information was not reliably reported for several players with the methodology used.

\begin{tabular}{|c|c|c|c|c|}
\hline & Sample size (n) & BMI $\left(\mathrm{kg} / \mathrm{m}^{2}\right)$ & Age at injury (y) & Playing experience (y) \\
\hline Overall & 55 & $22.7(1.80)$ & $25.8(4.01)$ & $8.4(4.01)$ \\
\hline Shoulder & 15 & $22.1(2.17)$ & $26.2(3.78)$ & 8.9 (3.74) \\
\hline Elbow & 15 & $23.0(1.09)$ & $26.0(4.98)$ & $8.3(5.01)$ \\
\hline Wrist & 13 & $22.3(1.87)$ & $25.4(3.34)$ & $8.2(3.02)$ \\
\hline Нiр & 12 & $23.7(1.64)$ & $25.4(4.05)$ & $8.1(4.33)$ \\
\hline Male & 39 & $23.5(1.03)^{\star}$ & $26.3(4.16)$ & $8.4(4.34)$ \\
\hline Female & 16 & $20.8(1.90)$ & $24.5(3.40)$ & $8.4(3.21)$ \\
\hline
\end{tabular}

\section{TABLE 1: Demographics of professional tennis players before surgery.}

Reported as “mean (standard deviation)", * $p=0.002$.

All of the shoulder and elbow procedures that met the inclusion criteria were performed on the players' dominant arms (shoulder: 12 right-handed, 3 left-handed; elbow: all right-handed). Eleven of the 13 wrist surgeries were on the players' non-dominant wrists (11 right-handed, 2 left-handed). Significantly, all players in the wrist cohort were known to utilize a two-handed backhand. Ten of the 12 hip surgeries were on the right hip, and all players in this cohort were right-handed. 


\section{Cureus}

The sole demographic difference was a significantly higher BMI in male players compared to female players $(\mathrm{p}<0.01)$ (Table 1). The average age at the time of surgery was $25.8( \pm 4)$ years, and the average professional career length before surgery was $8.4( \pm 4)$ years (Table 1$)$. There were no significant differences between joint cohorts in player age or career length before surgery $(\mathrm{p}=0.93$ and $\mathrm{p}=0.95$, respectively). Furthermore, there were no significant differences between cohorts in year-end singles ranking for the preoperative year (Table 2). Recovery time was significantly longer following shoulder surgery compared to the elbow, wrist, and hip surgery $(\mathrm{p}<0.01)$ (Table 3$)$.

\begin{tabular}{|c|c|c|c|}
\hline & Sample size (n) & Avg. ranking & SD \\
\hline Overall & 55 & 63 & 76 \\
\hline Shoulder & 15 & 72 & 71 \\
\hline Elbow & 15 & 44 & 44 \\
\hline Wrist & 13 & 56 & 70 \\
\hline Нip & 12 & 83 & 112 \\
\hline Male & 39 & 70 & 79 \\
\hline Female & 16 & 48 & 67 \\
\hline
\end{tabular}

TABLE 2: World ranking before surgery.

SD: standard deviation.

\begin{tabular}{|c|c|c|c|}
\hline & Sample size (n) & Avg. TIR (days) & SD \\
\hline Overall & 55 & 186 & 117 \\
\hline Shoulder & 15 & $279^{\star}$ & 100 \\
\hline Elbow & 15 & 142 & 117 \\
\hline Wrist & 13 & 148 & 18 \\
\hline Нір & 12 & 163 & 117 \\
\hline Male & 39 & 180 & 119 \\
\hline Female & 16 & 202 & 115 \\
\hline
\end{tabular}

TABLE 3: Time to return to sport following surgery.

TTR: time to return, * $p<0.01$. SD: standard deviation.

Athletes who underwent arthroscopic shoulder or elbow surgery had a significant decline in ranking after the first postoperative year of play ( $<0.01$ and $p=0.03$, respectively) (Figure 1 ). While the elbow cohort returned to baseline ranking in the second postoperative year, players who underwent arthroscopic shoulder surgery continued to have significantly lower rankings compared to the baseline $(\mathrm{p}<0.01)$. Furthermore, the shoulder-surgery cohort demonstrated a significantly greater decline in ranking compared to all other cohorts in both the first and second years postoperatively $(\mathrm{p}<0.01$ and $\mathrm{p}=0.01$, respectively) (Figure 1). From a performance-metric perspective, there was a significantly greater decrease in aces per service game in the shoulder cohort compared to all other cohorts in the first year postoperatively (shoulder: 0.20 acesper-game decrease; elbow: 0.01 aces-per-game decrease; wrist: 0.08 aces-per-game increase; hip: 0.03 acesper-game decrease; $\mathrm{p}=0.04)$. 


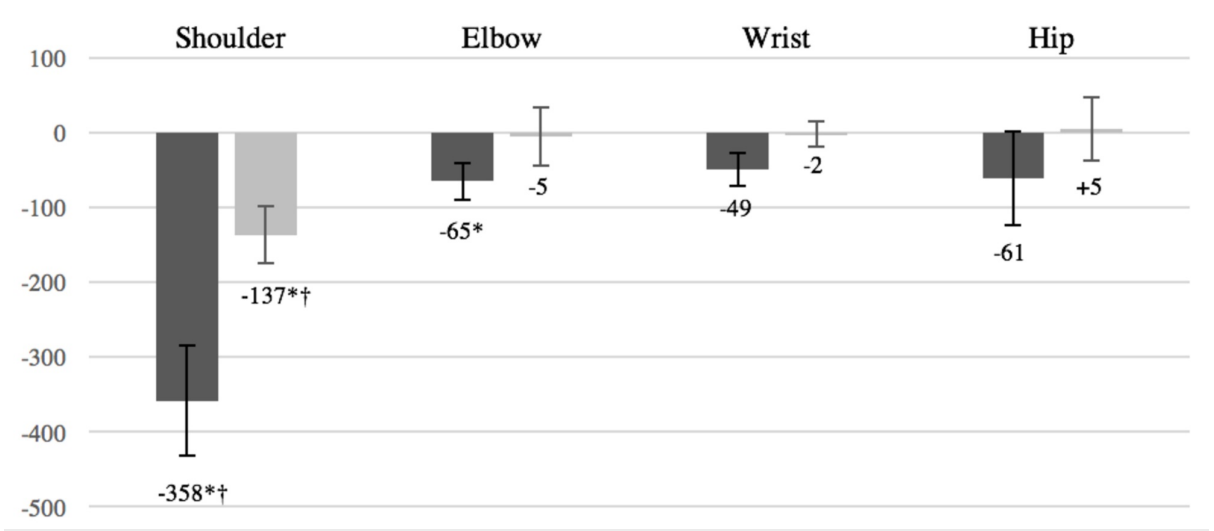

FIGURE 1: Change in ranking after surgery.

Dark gray: change in ranking from preinjury year to return year one.

Light gray: change in ranking from preinjury year to return year two.

After appropriate recovery time, athletes who underwent arthroscopic wrist or hip surgery demonstrated no significant change in ranking, at either time point. While every player in the shoulder cohort experienced a decline in ranking after the first postoperative year, a few players in each of the other cohorts showed improvement in the ranking (elbow: 4 players; wrist: 2 players; hip: 3 players).

Out of the 55 tennis players who underwent surgery, 30 are currently active in the professional circuit. Considering the data available for those who retired (shoulder, $n=6$; elbow, $n=6$; wrist, $n=6$; hip, $n=7$ ), the average career length after surgery was $5.7( \pm 2.6)$ years. And there was no significant variation on this metric depending on the location of surgery either (shoulder: 5.5 years; elbow: 6.2 years; wrist: 7.1 years; hip: 3.8 years; $\mathrm{p}=0.14$ ). There were no significant differences in any postoperative measures between genders.

\section{Discussion}

Tennis players faced with musculoskeletal injuries often undergo arthroscopic surgery with the goal of returning to their baseline level of performance. In comparison to recreational athletes, professional tennis players must return to a significantly more demanding level of play in a safe and timely manner. Since the impact of injury and the recovery time are much more critical in this patient group, athletes and their medical teams must be aware of the impact of surgical treatments on expected postoperative outcomes.

The data in our study suggest that professional tennis players who underwent arthroscopic shoulder surgery experienced the longest TTR and the greatest decline in postoperative performance. This is consistent with the data reported by Young et al., who found that arthroscopic shoulder surgery in female tennis players $(\mathrm{n}=$ 8) was associated with a prolonged and incomplete return to play [14]. There are a number of potential explanations for these findings. One explanation is that the shoulder is a critical joint in the production of all tennis strokes, especially the serve [21]. Significantly, all athletes in the shoulder cohort underwent surgery on their dominant arm. Furthermore, prior research in joint biomechanics has demonstrated that dominant-shoulder rotation is the greatest contributor to serve velocity in elite tennis players [22-24]. Thus, arthroscopic shoulder surgery may be associated with a reduction in the serve velocity, a critical component of performance in professional players. This is consistent with our results that male players in the shoulder cohort experienced a significantly greater drop in aces per service game compared to all other cohorts. The decline of ranking in the shoulder cohort may also be related to the longer TTR since time away from the sport could negatively impact performance and ranking.

Conversely, players who underwent elbow, wrist, and hip surgeries experienced significantly better outcomes. Athletes who underwent arthroscopic elbow surgery demonstrated a significant decline in ranking in their first postoperative year. However, while the shoulder cohort continued to have lower rankings after the second year of return, the elbow cohort returned to baseline performance levels after the second year. Of note, the majority of wrist operations were performed on the non-dominant wrist, which is consistent with previous literature reporting a higher prevalence of injuries in the non-dominant wrists of players with twohanded backhands [25]. Similarly, our findings in the hip cohort suggest that the hip on the dominant side is at a greater risk for injury and the need for surgical treatment. This may be due to the role of "back leg drive" in the initiation of trunk rotation for the serve and forehand, which exerts substantial stress on the dominant hip [21, 26]. 
The average age and career length before surgery were very similar across cohorts (Table 1). This finding may suggest that the pathology leading to arthroscopic treatment was a chronic, wear-and-tear mechanism as opposed to an acute injury leading to a structural lesion. Further studies may lead to training protocols designed to strengthen these joints prior to this time threshold in a professional tennis player's career, in order to avoid future surgical procedures.

Although this study utilized a previously published protocol, several important limitations remain. Details regarding injury diagnosis, radiographic findings, and exact procedure performed were not available for all players, which limited the conclusions we could make. We attempted to compensate for this limitation by utilizing each player as his/her own control, as well as by comparing cohorts as a whole. However, we were unable to make conclusions regarding the effect of the specific type of arthroscopic surgery. It is possible that the performance after debridement is different than that after repair. In addition, changes in ranking may have been influenced by a variety of other factors, including first-round exits, time off from surgery, and opponent skill level. Finally, it is possible that successful, newsworthy players had a higher chance of meeting the inclusion criteria given our methodology of using public archives. However, there was still a considerable gulf among the ranking of the players whose pre-surgery data we analyzed (Table 2), which indicates that the implications of this study are not limited to top-ranked players only.

\section{Conclusions}

Professional tennis players are predisposed to a variety of musculoskeletal injuries that may require arthroscopic surgery, including those of the shoulder, elbow, wrist, and hip. The results of this study suggest that arthroscopic shoulder surgery is associated with a prolonged TTR to play and a significant decline in ranking after the first and second years of return from surgery. To the best of our knowledge, this study represents the largest database of professional tennis players who have undergone arthroscopic surgery and may allow physicians to provide evidence-based recommendations about expectations after surgical treatment.

\section{Additional Information \\ Disclosures}

Human subjects: Consent was obtained by all participants in this study. Animal subjects: All authors have confirmed that this study did not involve animal subjects or tissue. Conflicts of interest: In compliance with the ICMJE uniform disclosure form, all authors declare the following: Payment/services info: All authors have declared that no financial support was received from any organization for the submitted work. Financial relationships: Matthew D Saltzman, MD declare(s) personal fees from CareFusion. Matthew D Saltzman, MD declare(s) personal fees from Medacta. Matthew D Saltzman, MD declare(s) personal fees and royalties from Wright Medical Technology, Inc. Other relationships: All authors have declared that there are no other relationships or activities that could appear to have influenced the submitted work.

\section{References}

1. Gescheit DT, Cormack SJ, Duffield R, Kovalchik S, Wood TO, Omizzolo M, Reid M: Injury epidemiology of tennis players at the 2011-2016 Australian Open Grand Slam. Br J Sports Med. 2017, 51:1289-94. 10.1136/bjsports-2016-097283

2. McCurdie I, Smith S, Bell PH, Batt ME: Tennis injury data from The Championships, Wimbledon, from 2003 to 2012. Br J Sports Med. 2017, 51:607-11. 10.1136/bjsports-2015-095552

3. Sell K, Hainline B, Yorio M, Kovacs M: Injury trend analysis from the US Open Tennis Championships between 1994 and 2009. Br J Sports Med. 2014, 48:546-51. 10.1136/bjsports-2012-091175

4. Miller S: Modern tennis rackets, balls, and surfaces. Br J Sports Med. 2006, 40:401-5. 10.1136/bjsm.2005.023283

5. Hennig EM: Influence of racket properties on injuries and performance in tennis . Exerc Sport Sci Rev. 2007, 35:62-6. 10.1249/JES.0b013e31803ec43e

6. Dines JS, Bedi A, Williams PN, et al.: Tennis injuries: epidemiology, pathophysiology, and treatment. J Am Acad Orthop Surg. 2015, 23:181-9. 10.5435/JAAOS-D-13-00148

7. Sonnery-Cottet B, Edwards TB, Noel E, Walch G: Rotator cuff tears in middle-aged tennis players: results of surgical treatment. Am J Sports Med. 2002, 30:558-64. 10.1177/03635465020300041601

8. Bigiliani LU, Kimmel J, McCann PD, Wolfe I: Repair of rotator cuff tears in tennis players . Am J Sports Med. 1992, 20:112-7. 10.1177/036354659202000203

9. Neuman BJ, Boisvert CB, Reiter B, Lawson K, Ciccotti MG, Cohen SB: Results of arthroscopic repair of type II superior labral anterior posterior lesions in overhead athletes: assessment of return to preinjury playing level and satisfaction. Am J Sports Med. 2011, 39:1883-8. 10.1177/0363546511412317

10. Neri BR, ElAttrache NS, Owsley KC, Mohr K, Yocum LA: Outcome of type II superior labral anterior posterior repairs in elite overhead athletes: effect of concomitant partial-thickness rotator cuff tears. Am J Sports Med. 2011, 39:114-20. 10.1177/0363546510379971

11. Sciascia A, Myers N, Kibler WB, Uhl TL: Return to preinjury levels of participation after superior labral repair in overhead athletes: a systematic review. J Athl Train. 2015, 50:767-77. 10.4085/1062-6050-50.3.06

12. Klouche S, Lefevre N, Herman S, Gerometta A, Bohu Y: Return to sport after rotator cuff tear repair: a systematic review and meta-analysis. Am J Sports Med. 2016, 44:1877-87. 10.1177/0363546515598995

13. Philippon MJ, McNamara SC, Briggs KK: Outcomes for femoroacetabular impingement with chondrolabral dysfunction in professional tennis players following hip arthroscopy. Journal of Medicine and Science in 
Tennis. 2016, 21:6-9.

14. Young SW, Dakic J, Stroia K, Nguyen ML, Safran MR: Arthroscopic shoulder surgery in female professional tennis players: ability and timing to return to play. Clin J Sport Med. 2017, 27:357-60.

10.1097//SM.0000000000000361

15. Hsu WK, McCarthy KJ, Savage JW, et al.: The Professional Athlete Spine Initiative: outcomes after lumbar disc herniation in 342 elite professional athletes. Spine J. 2011, 11:180-6. 10.1016/j.spinee.2010.12.009

16. Kester BS, Behery OA, Minhas SV, Hsu WK: Athletic performance and career longevity following anterior cruciate ligament reconstruction in the National Basketball Association. Knee Surg Sports Traumatol Arthrosc. 2017, 25:3031-37. 10.1007/s00167-016-4060-y

17. Mai HT, Alvarez AP, Freshman RD, et al.: The NFL Orthopaedic Surgery Outcomes Database (NO-SOD): the effect of common orthopaedic procedures on football careers. Am J Sports Med. 2016, 44:2255-62. $10.1177 / 0363546516651426$

18. Minhas SV, Kester BS, Hsu WK: Outcomes after lumbar disc herniation in the National Basketball Association. Sports Health. 2016, 8:43-9. 10.1177/1941738115608361

19. Minhas SV, Kester BS, Larkin KE, Hsu WK: The effect of an orthopaedic surgical procedure in the National Basketball Association. Am J Sports Med. 2016, 44:1056-61. 10.1177/0363546515623028

20. Mai HT, Chun DS, Schneider AD, et al.: Performance-based outcomes after anterior cruciate ligament reconstruction in professional athletes differ between sports. Am J Sports Med. 2017, 45:2226-32. $10.1177 / 0363546517704834$

21. Elliott B: Biomechanics and tennis. Br J Sports Med. 2006, 40:392-96. 10.1136/bjsm.2005.023150

22. Cohen DB, Mont MA, Campbell KR, Vogelstein BN, Loewy JW: Upper extremity physical factors affecting tennis serve velocity. Am J Sports Med. 1994, 22:746-50. 10.1177/036354659402200604

23. Gordon BJ, Dapena J: Contributions of joint rotations to racquet speed in the tennis serve .J Sports Sci. 2006, 24:31-49. 10.1080/02640410400022045

24. Elliott BC, Marshall RN, Noffal GJ: Contributions of upper limb segment rotations during the power serve in tennis. J Appl Biomech. 1995, 11:433-42.

25. Montalvan B, Parier J, Brasseur JL, Le Viet D, Drape JL: Extensor carpi ulnaris injuries in tennis players: a study of 28 cases. Br J Sports Med. 2006, 40:424-29. 10.1136/bjsm.2005.023275

26. Elliott BC: Biomechanics of the serve in tennis . Sports Med. 1988, 6:285-94. 10.2165/00007256-19880605000004 